\title{
КЛАССИФИКАЦИЯ ВОЗМОЖНЫХ ЗАДАЧ ДЛЯ ИССЛЕДОВАНИЯ НЕЛИНЕЙНЫХ ВОЛН В НАСЫЩЕННЫХ ПОРИСТЫХ СРЕДАХ
}

\author{
Керимова Ш.А. \\ Стариий научный сотрудник \\ Институт математики и механики НАН Азербайджана \\ Республика Азербайджан, г.Баку
}

Аннотация. Применение вычислительной техники и современных компьютерных технологий для анализа состояния гидроэкосистем открывает новые возможности как перед учеными-исследователями, так и перед лицами и организациями, принимающими ответственные решения по рациональному использованию природной среды на благо человека, а также поддержки решений в отношении осуществления мероприятий во всех видах хозяйственной и культурно-бытовой деятельности с использованием водных ресурсов и водосборных бассейнов путем формирования высококвалифицированных рекомендаций [1].

Abstarct. The use of computer technology and modern computer technologies for analyzing the state of hydroecosystems opens up new opportunities both for scientists-researchers and for persons and organizations making responsible decisions on the rational use of the natural environment for the benefit of humans, as well as support for decisions regarding the implementation of measures in all types economic and cultural and household activities using water resources and drainage basins by forming highly qualified recommendations [1].

Ключевые слова: классификация, деформация, упруго, еластическая, пластическая, движения.

Key words: classification, deformation, elastic, elastic, plastic, motion.

При изучении различных конкретных задач возможно значительное упрощение этой системы, поэтому желательно иметь возможность по характеру рассматриваемой конкретной задачи установить, какие упрощения в этом случае возможны и какой упрощенной системой уравнений следует пользоваться. Ниже дается классификация возможных задач для отмеченной системы уравнений с выводом упрощенных систем для каждого из классов задач.

Уравнения из [2,3] имеют вид

$$
\begin{gathered}
\rho\left(\frac{\partial v_{i}}{\partial t}+v_{j} \frac{\partial v_{i}}{\partial x_{j}}\right)=\rho F_{i}^{e}-\frac{\partial p}{\partial x_{j}}+\frac{\partial S_{i j}}{\partial x_{j}}, \\
\frac{\partial \rho}{\partial t}+\frac{\partial\left(\rho v_{i}\right)}{\partial x_{i}}=0, p=f\left(\theta, \theta_{*}\right) \theta \equiv 1-\frac{\rho_{0}}{\rho}, \theta_{*} \equiv 1-\frac{\rho_{0}}{\rho} \\
\frac{\partial \theta_{*}}{\partial t}+v_{i} \frac{\partial \theta_{*}}{\partial x_{i}}=\left(\frac{\partial \theta}{\partial t}+v_{i} \frac{\partial \theta}{\partial x_{i}}\right) e\left(\theta-\theta_{*}\right) e\left(\frac{\partial \theta}{\partial t}+v_{i} \frac{\partial \theta}{\partial x_{j}}\right) \\
G=\left(\frac{\partial v_{i}}{\partial x_{j}}+v_{j} \frac{\partial v_{j}}{\partial x_{i}}-\frac{2}{3} \frac{\partial v_{k}}{\partial x_{k}} \delta_{i j}\right)=\frac{D S_{i j}}{D t}+\lambda S_{i j} \\
\frac{D S_{i j}}{D t}=\frac{\partial S_{i j}}{\partial t}+v_{k} \frac{\partial S_{i j}}{\partial x_{k}}-\Omega_{i j} S_{j k}-\Omega_{j k} S_{i k}, \Omega_{i j}=\frac{1}{2}\left(\frac{\partial v_{i}}{\partial x_{j}}-\frac{\partial v_{k}}{\partial x_{i}}\right) \\
\lambda=\frac{2 G W-F^{\prime}(p)\left(\partial p / \partial t+v_{i} \partial p / \partial x_{i}\right)}{2 F(p)} e\left[I_{2}-F(p)\right] e\left[2 G W-F^{\prime}(p)\left(\frac{\partial p}{\partial t}+v_{i} \frac{\partial p}{\partial x_{i}}\right)\right] \\
W \equiv \frac{1}{2} S_{i j}\left(\frac{\partial v_{i}}{\partial x_{j}}-\frac{\partial v_{j}}{\partial x_{i}}\right), J \equiv \frac{1}{2} S_{i j} S_{i j}
\end{gathered}
$$

В этих уравнениях фигурируют определяемые из опыта функции- характеристики грунта

$$
p=f\left(\theta, \theta_{*}\right), I_{2}=F(p), G=G\left(\theta_{*}\right)(1.2)
$$

Способ определения этих функций из динамических и статических опытов указан в работах [2,3], а некоторые результаты такого определения содержатся в работах[4,5].

Основная задача механики грунтов, возникающая для системы (1.1), состоит в следующем. Дана область $\Omega$, заполненная средой, находящейся сначала в покое. На части границы этой области заданы напряжения $P_{n}$ на остальной части границы - перемещения и как функции координат точек границы и времени. Требуется определить возникающие в среде движения и распределение напряжений.

Задание граничных функций $P_{n}$ и $u$ всегда может быть представлено в виде 


$$
P_{n}=\sigma_{0} \Pi_{n}\left(\frac{x_{i}}{l}, \frac{t}{t_{0}}\right), u=V_{0} t_{0} U\left(\frac{x_{i}}{l}, \frac{t}{t_{0}}\right)
$$

где $\Pi_{n}$ и $U$-безразмерные функции безразмерных аргументов, поэтому граничные условия вносят в математическую формулировку задачи параметры $\sigma_{0}, V_{0}, t_{0}, l$ - характерные значения напряжений, скоростей, времени и линейных размеров. Различные классы задач будут при прочих одинаковых условиях определяться различием в численных значениях этих параметров. Для того чтобы произвести классификацию задач, необходимо эти параметры дополнить параметрами, содержащимися в системе уравнений (1.1). Для выявления этих параметров заметим, что соотношения (1.2) всегда можно представить в виде

$$
\begin{aligned}
& p=K f\left(\theta, \theta_{*}\right), I_{2}=\sigma_{*}^{2} F\left(p / \sigma_{*}\right), G=G_{0} g\left(\theta_{*}\right) \\
& \underset{\substack{\theta \rightarrow 0 \\
\theta_{*} \rightarrow \min \theta_{*}}}{f\left(\theta, \theta_{*}\right)} \rightarrow \underset{p / \sigma_{*} \rightarrow 0}{F\left(p / \sigma_{*}\right)} \rightarrow 1, \underset{\theta_{*} \rightarrow \min \theta_{*}}{g\left(\theta_{*}\right) \rightarrow 1} \rightarrow 1
\end{aligned}
$$

Введем обозначение

$$
S_{\infty}=\lim _{p / \sigma_{*} \rightarrow \infty} \sqrt{\sigma_{*}^{2} F\left(p / \sigma_{*}\right)}=\sigma_{*} \sqrt{F(\infty)}
$$

Опыт показывает, что

$$
K \sim G_{0}, \sigma_{*}<<G_{0}
$$

Кроме того, можно считать, что $S_{\infty}$ имеет порядок $G_{0}$ или меньше, т.е.

$$
S_{\infty} \leq G_{0}
$$

Таким образом, в число параметров следует включить еще $K, G_{0}, \sigma_{*}, S_{\infty}, \rho_{0}$, таким что полная система существенных постоянных определяющих параметров задачи приобретает вид

$$
\sigma_{0}, V_{0}, t_{0}, l, K, G_{0}, \sigma_{*}, S_{\infty}, \rho_{0}
$$

Отметим, что в случаях, когда граничные условия даны только в напряжениях или только в перемещениях, в эту систему будет входить либо только $\sigma_{0}$, либо только $V_{0}$, а $\sigma_{0}$ и $V_{0}$ будут связаны некоторым соотношением. Классификация будет в конечном счете сводиться к установлению некоторых оценок и неравенств для безразмерных комбинаций, составленных из (1.8), при выполнении которых будут место соответствующие упрощения в системе (1.1).

2. Начнем рассмотрение с чисто упругого случая, когда $\sigma_{0}<\sigma_{*}$ и $u_{0} \sim V_{0} t_{0}$ весьма малы. В этом случае $\lambda \equiv 0$ в уравнениях (1.1), и соотношение между девиаторами тензоров напряжений и скоростей деформаций из системы (1.1) принимает вид

$$
G=\left(\frac{\partial v_{i}}{\partial x_{j}}+\frac{\partial v_{j}}{\partial x_{i}}-\frac{2}{3} \frac{\partial v_{k}}{\partial x_{k}} \delta_{i j}\right)=\frac{D S_{i j}}{D t}
$$

Произведем оценку различных членов в этом соотношения, полагая, что

$$
v_{i}=V_{0} V_{i}\left(\frac{x_{k}}{l}, \frac{t}{t_{0}}\right), S_{i j}=\sigma_{0} \sum_{i j}\left(\frac{x_{k}}{l}, \frac{t}{t_{0}}\right)
$$

причем, функции $V_{i}, \sum_{i j}$ и их производные по безразмерным аргументам имеют порядок единицы. Будем иметь из (2.1)

$$
G_{0} \frac{V_{0}}{l} \sim \sigma_{0}\left(\frac{1}{t_{0}}+\frac{V_{0}}{l}\right)
$$

Так как рассматриваемом случае $\sigma_{0} \sim \sigma_{*}<<G_{0}$, из (2.3) следует, что 


$$
\frac{V_{0} t_{0}}{l} \sim, \frac{\sigma_{0}}{G_{0}}<<1
$$

Это означает, что смешения $u_{0} \sim V_{0} t_{0}$ и деформации $u_{0} / l$ малы, а в выражениях для полных производных по времени можно пренебречь конвективными членами,

$$
\frac{D}{D t} \sim \frac{d}{d t} \equiv \frac{\partial}{\partial t}+v_{i} \frac{\partial}{\partial x_{i}} \sim \frac{\partial}{\partial t}
$$

Система (1.1) переходит при этом в

$$
\begin{aligned}
\rho_{0} \frac{\partial v_{i}}{\partial t} & =\rho_{0} F_{i}^{e}-\frac{\partial p}{\partial x_{i}}+\frac{\partial s_{i j}}{\partial x_{j}}, \frac{\partial \theta}{\partial t}+\frac{\partial v_{i}}{\partial x_{i}}=0 \\
p & =K \theta, G_{0}\left(\frac{\partial v_{i}}{\partial x_{i}}+\frac{\partial v_{j}}{\partial x_{i}}-\frac{2}{3} \frac{\partial v_{k}}{\partial x_{k}} \delta_{i j}\right)=\frac{\partial s_{i j}}{\partial t}
\end{aligned}
$$

Второе и четвертое из этих уравнений интегрируются и вместе с третьим дают закон Гука

$$
S_{i j}=G_{0}\left(\frac{\partial u_{i}}{\partial x_{j}}+\frac{\partial u_{j}}{\partial x_{i}}+\frac{2}{3} \theta \delta_{i j}\right), \theta=-\frac{\partial u_{k}}{\partial x_{k}}, p=K \theta\left(u_{i}=\int_{0}^{t} v_{i} d t\right)
$$

где $u_{i}$-смещение. Таким образом, для этого случая система (1.1) переходит в обычные уравнения линейной теории упругости.

Для уравнений (2.6) интересно рассмотреть два класса движений; динамических и квазистатических. В первом случае все члены в уравнении импульсов имеют один порядок, т.е.

$$
\rho_{0} \frac{V_{0}}{t_{0}} \sim \frac{\sigma_{0}}{l}
$$

Сравнивая это с (2.4), получаем

$$
\frac{l}{t_{0}} \sim \sqrt{\frac{G_{0}}{\rho_{0}}} \sim C, \sigma_{0} \sim \rho_{0} V_{0} C
$$

и так как $\sigma_{0} \sim K \theta \sim G_{0} \theta$ то и $V_{0} \sim \theta C$. В этих оценках с- характерная скорость распространения упругих волн. Все это -известные соотношения для упругих волн. В случае, когда движение квазистатическое, должно быть

$$
\rho_{0} \frac{V_{0}}{t_{0}}<<\frac{\sigma_{0}}{l} \text { или } \frac{l}{t_{0}}<<\sqrt{\frac{G_{0}}{\rho_{0}}} \sim C
$$

Это условие определяет масштаб времени $t_{0}$ граничных функций (1.3) при котором упругими волнами можно пренебречь. Если ввести «волновой» масштаб времени $t_{\omega} \sim l / C$, то условие (2.9) запишется в виде $t_{0}>>t_{\omega}$.

3. Рассмотрим движение с упругопластическими, но малыми деформациями. Здесь, как и в предыдущем случае, $\sigma_{0} \sim \sigma_{*}, u_{0} \sim V_{0} t_{0}<<l$, но $\lambda \neq 0$. Очевидно, оценка $(2,5)$ сохраняются. Оценка членов в законе упругопластического течения

$$
G=\left(\frac{\partial v_{i}}{\partial x_{j}}+\frac{\partial v_{j}}{\partial x_{i}}-\frac{2}{3} \frac{\partial v_{k}}{\partial x_{k}} \delta_{i j}\right)=\frac{D S_{i j}}{D t}+\lambda S_{i j}, \text { дает } G_{0} \frac{V_{0}}{l} \sim \frac{\sigma_{0}}{t_{0}}+\left(\frac{G_{0} V_{0}}{\sigma_{0} l}+\frac{1}{t_{0}}\right) \sigma_{0}
$$

В правой части этого соотношения все члены должны быть одного порядка (упругие и пластические составляющие деформации одного порядка в рассматриваемом случае). Это возможно, если

$$
\frac{G_{0} V_{0}}{\sigma_{0} l} \sim 1(3.2)
$$

Выполнение условия(3.2) одновременно приводит и к удовлетворению условия (3.1) и, кроме того, это условие сводится к (2.4). Условия динамичности и вытекающие из них оценки для $\sigma_{0}$ и $V_{0}$, а также условие квазистатичности в этом случае также сохраняются теми же, что в предыдущем случае. Система (1.1) переходит в следующее уравнение: 


$$
\begin{gathered}
\rho_{0} \frac{\partial v_{i}}{\partial t}=\rho_{0} F_{i}^{e}-\frac{\partial p}{\partial x_{i}}+\frac{\partial S_{i j}}{\partial x_{j}}, p=f\left(\theta, \theta_{*}\right) \frac{\partial \theta}{\partial t}+\frac{\partial v_{i}}{\partial t}=0, \frac{\partial \theta_{*}}{\partial t}=\frac{\partial \theta}{\partial t} e\left(\theta-\theta_{*}\right) e \frac{\partial \theta}{\partial t} \\
G_{0}=\left(\frac{\partial v_{i}}{\partial x_{j}}+\frac{\partial v_{j}}{\partial x_{i}}-\frac{2}{3} \frac{\partial v_{k}}{\partial x_{k}} \delta_{i j}\right)=\frac{D S_{i j}}{D t}+\lambda S_{i j} \\
\lambda=\frac{2 G_{0} W-F^{\prime}(p) \partial p / \partial t}{2 F(p)} e\left[I_{2}-F(p)\right] e\left[2 G_{0} W-F^{\prime}(p) \frac{\partial p}{\partial t}\right]
\end{gathered}
$$

4. Рассмотрим теперь движения с большими упруго-пластическими деформациями, но при умеренных напряжениях, т.е. случай, когда $V_{0} t_{0} \sim l$ и $\sigma_{*}<<\sigma_{0}<<G_{0}$. Это реальный и практически интересный случай, так как, например, для песка $\sigma_{*} \sim 0,5 \kappa \Gamma / \mathrm{cm}^{2}, G_{0} \sim 10^{3} \kappa \Gamma / \mathrm{cm}^{2}$ и, следовательно, речь идет о напряжениях $\sigma_{0} \sim 10 \kappa \Gamma / \mathrm{cm}^{2}-100 \kappa \Gamma / \mathrm{cm}^{2}$. В этом случае

$$
\frac{G_{0} V_{0} t_{0}}{\sigma_{0} l}>>1
$$

и из соотношения (3.1) (закон течения) следует, что упругими составляющими в законе течения можно пренебречь. Уравнение неразрывности переходит в оценочное соотношение $\frac{\theta}{t_{0}}+\frac{V_{0}}{l} \sim 0$, Опыт показывает, что при $\rho \sim \sigma_{0} \sim 10 \kappa \Gamma / \mathrm{cm}^{2}-100 \kappa \Gamma / \mathrm{cm}^{2}, \theta \sim 10^{-2}$. Отсюда и из последнего соотношения следует, что в уравнении неразрывности членами, содержащими производные плотности, следует пренебречь. Наконец, оценка членов в уравнениях импульсов для динамической задачи дает

$$
\rho_{0}\left(\frac{V_{0}}{t_{0}}+\frac{V_{0}^{2}}{l}\right) \sim \frac{\sigma_{0}}{l} \text { или } \sigma_{0} \sim \rho_{0} V_{0}^{2}
$$

поэтому уравнения эти должны сохраниться в неизменном виде (с заменой $\rho$ на $\rho_{0}$, конечно).

Таким образом, в этом случае из системы (1.1) получаем

$$
\begin{array}{r}
\rho_{0}\left(\frac{\partial v_{i}}{\partial t}+v_{i} \frac{\partial v_{i}}{\partial x_{j}}\right)=\rho_{0} F_{i}^{e}-\frac{\partial p}{\partial x_{i}}+\frac{\partial s_{i j}}{\partial x_{j}}, \frac{\partial v_{i}}{\partial x_{i}}=0 \\
\frac{\partial v_{i}}{\partial x_{j}}+\frac{\partial v_{j}}{\partial x_{i}}=\frac{W}{F(p)} e\left[I_{2}-F(p)\right] e(W) S_{i j}
\end{array}
$$

Отметим, что после того, как из (4.3) найдено распределение давления $p$, задача отыскания распределения плотностей может быть решена отдельно при помощи зависимости $p=f=\left(\theta, \theta_{*}\right)$ и функции $\partial p / \partial t+v_{i} \partial p / \partial x_{i}$. В рассматриваемом случае условие динамичности движения дает

$$
\frac{l}{l_{0}} \sim \sqrt{\frac{\sigma_{0}}{P_{0}}} \sim \sqrt{\frac{\sigma_{0}}{G_{0}}} C \sim \sqrt{\theta} C \text { или } t_{0} \sim \frac{1}{\sqrt{\theta}} t_{\omega}
$$

Сказанное в этом пункте, относится только к областям движения, удаленным от фронтов волн. В областях, примыкающих к таким фронтам, дело будет обстоять, как в предыдущем случае, так как из условий совместности на фронте волны следуют оценки для прифронтовых областей движения $t_{0} \sim t_{\omega}, V_{0} \sim \theta C, \sigma_{0} \sim \rho_{0} \theta C^{2}$. Таким образом, в прифронтовых областях движение будет описываться системой (3.3). Следует только иметь в виду, что в задачах, для которых в прифронтовой области градиенты велики и волна распространяется на расстояния, значительные по сравнению с ее длиной (короткая волна), нужно учитывать конвективные члены в производных по времени [5]. Но и в этом случае основные уравнения значительно упрощаются при помощи соображений из работы [5] и допускают сравнительно простой анализ, который здесь однако не будет проводиться. В рассматриваемом случае возможны два типа динамических движений среды: в прифронтовой зоне, где движение носит резко выражений волновой характер, и вдали от фронта, где волновые эффекты пренебрежимо, и движение описывается в схеме несжимаемой жидкости. Надо отметить, что такого рода ситуация имеет место при описании движений капельных жидкостей (воды, например), которые тоже обладают весьма малой сжимаемостью. Очевидно, причина такого разделения и в случае капельной жидкости, в рассматриваемом здесь случае грунта одна - это малая сжимаемость среды. Объединенная система имеет вид Это система отличается от системы для прифронтовой области только наличием конвективных слагаемых в полной производной скорости по времени, но эти слагаемые там малы, и фактически система не отличается от системы (3.3) для прифронтовой области. 


$$
\begin{gathered}
\rho_{0}\left(\frac{\partial v_{i}}{\partial t}+v_{j} \frac{\partial v_{i}}{\partial x_{j}}\right)=\rho_{0} F_{i}^{e}-\frac{\partial p}{\partial x_{j}}+\frac{\partial S_{i j}}{\partial x_{j}}, p=f\left(\theta, \theta_{*}\right) \\
\frac{\partial \theta}{\partial t}+\frac{\partial v_{i}}{\partial x_{i}}=0, \frac{\partial \theta_{*}}{\partial t}=\frac{\partial \theta}{\partial t} e\left(\theta-\theta_{*}\right) e\left(\frac{\partial \theta}{\partial t}\right) \\
G=\left(\frac{\partial v_{i}}{\partial x_{j}}+\frac{\partial v_{j}}{\partial x_{i}}-\frac{2}{3} \frac{\partial v_{k}}{\partial x_{k}} \delta_{i j}\right)=\frac{\partial S_{i j}}{\partial t}+\lambda S_{i j} \\
\lambda=\frac{2 G W-F^{\prime}(p) \partial p / \partial t}{2 F(p)} e\left[I_{2}-F(p)\right] e\left[2 G W-F^{\prime}(p) \frac{\partial p}{\partial x_{i}}\right]
\end{gathered}
$$

С другой стороны, система (4.5) в области, удаленной от фронта, почти не отличается от системы (4.3) так как в этой области производные по времени во всех уравнениях, кроме уравнений импульсов, пренебрежимо малы. Условием квазистатичности в рассматриваемом случае, будет (см. (4.4)

$$
t_{0}>>\frac{t_{\omega}}{\sqrt{\theta}}>>t_{\omega}
$$

т.е. здесь имеем более сильное условие, чем в предыдущих случаях, когда было достаточно условия (2.10). Отметим, что для $\sigma_{0} \sim 10$ кГ $/ \mathrm{cm}^{2}-100$ кГ $/ \mathrm{cm}^{2}$ величина, $\theta \sim 10^{-2}$ и при $l \sim 10$ ми $C \sim 10^{2}$ м/секбудем иметь $t_{\omega} / \sqrt{\theta} \sim 1$ т.е. условие квазистатичности (4.6) требует, чтобы было $t_{0}>>1$ сек. Таким образом, для всех задач обычной строительной механики оснований и фундаментов это условие всегда выполнено. Лишь для взрывных и ударных явлений следует прибегать к динамическим уравнениям. При выполнении условия (4.6) основные уравнения получим:

$$
\begin{aligned}
-\frac{\partial p}{\partial x_{i}}+\frac{\partial S_{i j}}{\partial \omega_{j}}+\rho_{0} F_{i}^{e}=0, \frac{\partial v_{i}}{\partial x_{i}}=0 \\
\frac{\partial v_{i}}{\partial x_{j}}+\frac{\partial v_{j}}{\partial x_{i}}=\frac{W}{F(p)} e\left[I_{2}-F(p)\right] e(W) S_{i j}
\end{aligned}
$$

Это - уравнения жесткопластического квазистатического течения грунта с развитыми пластическими деформациями или уравнения «предельного равновесия среды» среды. В частном случае плоской задачи отсюда получаются уравнения плоского предельного равновесия статики сыпучей среды [7]. В самом далее, из закона получаем $S_{z z}=0$, что приводит условие пластичности к виду $\frac{1}{2}\left[\left(\sigma_{x x}+p\right)^{2}+\left(\sigma_{y y}+p\right)^{2}+2{\sigma_{x y}}^{2}\right]=F(p)$ а также приводит к соотношению $\sigma_{z z}=-$ рили $p=-\frac{1}{2}\left(\sigma_{x x}-\sigma_{y y}\right)$.

Это, в свою очередь, приводит условие пластичности и окончательному виду $\frac{1}{4}\left[\left(\sigma_{x x}-\sigma_{y y}\right)^{2}+4 \sigma_{x y}{ }^{2}\right]=$ $F\left[-\frac{1}{2}\left(\sigma_{x x}+\sigma_{y y}\right)\right]$. В случае, если $F(p)=(k p+b)^{2}$ например, работы[3,4], отсюда получаем

$\frac{1}{4}\left[\left(\sigma_{x x}-\sigma_{y y}\right)^{2}+4{\sigma_{x y}}^{2}\right]=\frac{k^{2}}{4}\left[-\left(\sigma_{x x}+\sigma_{y y}\right)+2 \frac{b}{k}\right]^{2}$. Условие предельного равновесия имеет вид $\frac{1}{4}\left[\left(\sigma_{x x}-\right.\right.$ $\left.\left.\sigma_{y y}\right)^{2}+4{\sigma_{x y}}^{2}\right]=\frac{\sin ^{2} \rho}{4}\left[-\left(\sigma_{x x}+\sigma_{y y}\right)+2 H\right]^{2}$, где $\rho-$ угол внутреннего трения, $H-$ сцепление. С точностью до обозначений и наименований эти условия совладают. Добавив к этому соотношению уравнения равновесия

$$
\frac{\partial \sigma_{x x}}{\partial x}+\frac{\partial \sigma_{x y}}{\partial y}+\rho_{0} F_{x}^{e}=0, \frac{\partial \sigma_{x y}}{\partial x}+\frac{\partial \sigma_{y y}}{\partial y}+\rho_{0} F_{x}^{e}=0
$$

Получаем систему трех уравнений для $\sigma_{x x}, \sigma_{x y}, \sigma_{y y}$, которая и составляет

единственную основу статики сыпучей среды [7].. $\quad$ Эти $\quad$ уравнения недос-таточно для естественной постановки и правильного решения задач статики сыпучей среды даже для плоского случая (не говоря о том, что статики сыпучей среды вообще не имеет уравнений для пространственной задачи).

5. Следующим будет случай, когда и упругопластические деформации велики, т.е. $\Gamma_{0} t_{0} \sim l$, и напряжения велики, т.е. Если при этом $S_{\infty}<<G_{0}$, то это будет предыдущий случай. Если же $S_{\infty} \sim G_{0}$, то никаких упрощений в уравнениях (1.1) уже сделать нельзя, так как при этом величина $\theta$ не будет мала: $\theta \sim 1$. Условие квазистатичности сохраняется в виде (4.6). В случае, когда имеется течение с очень большими напряжениями $\sigma_{0}>>S_{\infty}$, в силу 
того, что $S_{i j} \sim S_{\infty}<<\sigma_{0} \sim p$, всюду можно пренебречь касательными напряжениями, и система (1.1) перейдет в уравнения для идеальной сжимаемой жидкости с возможной необратимостью в объемных деформациях

$$
\begin{gathered}
\rho\left(\frac{\partial v_{i}}{\partial t}+v_{j} \frac{\partial v_{i}}{\partial x_{j}}\right)=\rho F_{i}^{e}-\frac{\partial p}{\partial x_{j}}, p=f\left(\theta, \theta_{*}\right) \\
\theta=1-\frac{\rho_{0}}{\rho} \theta_{*}=1-\frac{\rho_{0}}{\rho_{*}}, \frac{\partial p}{\partial t}+\frac{\partial\left(\rho v_{i}\right)}{\partial x_{i}}=0 \\
\frac{\partial \theta_{*}}{\partial t}+v_{i} \frac{\partial \theta_{*}}{\partial x_{i}}=\left(\frac{\partial \theta}{\partial t}+v_{i} \frac{\partial \theta}{\partial x_{i}}\right) e\left(\theta-\theta_{*}\right) e\left(\frac{\partial \theta}{\partial t}+v_{i} \frac{\partial \theta}{\partial x_{j}}\right)
\end{gathered}
$$

Рассмотрение квазистатических движений в этом случае неинтересно, так как они здесь тривиальны. Этим исчерпывается изучение всех возможных существенно разных типов движений грунта. Очевидно, аналогичный анализ можно произвести для любою другой твердой среды (для пластичных металлов, например), и результаты будут вполне подобны полученным выше.

1. Ш.А.Керимова, $\quad$ Ф.А.Сейфуллаев $\quad$ "Пространственно-трехмерная $\quad$ постановка задачи гидротермодинамики водоемов" // Актуальная наука"(1/(30)-2020) c.7-11

2. Николаевски В.Н., Бондарев Е.А., Миркин М.И. и другие. Движение смеси углеводорода в пористой среде. М.: "Nedra", 1968. 192p

3. Розеньберг М.Д., Кундин С.А., Курбанов А.К и другие. «Фильтрация жидкости и других многокомпонентных смесей в нефтяных стратах». М.: "Nedra", 1969, 456p

4. Слеттери Дж.С. . Теория перемещения импульса, энергии и массы в континумах. М.: Энергия, $1978,448 \mathrm{p}$

5. Тсибульски Г.Р. Уравнение неравновесия двухфазных фильтрация. В книге: Числовое решение проблемы фильтрования многофазный несжимаемой жидкости. Новосибирск, 1977, pp.203-213

6. Николаевски В.Н.. Геомеханика и жидкая динамика. М.: "Nedra", 1996

7. Хаазе Р. Термодинамика необратимых процессов. М.: Мир, 1967. 544c 